\title{
Mediastinal Hydatid Cyst Mimicking Malignant Mediastinal Neurogenic Tumor
}

\author{
Aram Baram, Fahmi H. Kakamad, Ali. A. Alawan \\ University of Sulaimani, Sulaimani, Iraq \\ Email: aram.baramm@gmail.com, drkakamad@gmail.com, alialwan2003@yahoo.com
}

Received 31 August 2013; revised 1 October 2013; accepted 8 October 2013

Copyright (C) 2014 by authors and Scientific Research Publishing Inc.

This work is licensed under the Creative Commons Attribution International License (CC BY). http://creativecommons.org/licenses/by/4.0/

(c) (i) Open Access

\begin{abstract}
Hydatid disease is caused by Echinococcus granulosus parasite. It is an endemic disease; particularly in many Mediterranean countries. The liver and the lungs are most frequently involved. Bone involvement is reported in $1 \%-2 \%$ of the cases and about $50 \%$ of those are seen in the spine. Herein we report a case of primary spinal extradural hydatid cyst that caused paraplegia due to compression of the dorsal spinal cord and was diagnosed initially as case of lumber prolapsed intervertebral disc. The cyst was only discovered when the patient had progressive paraplegia and the preoperative provisional diagnosis was posterior mediastinal neurogenic tumor causing destruction of the pedicle and lamina of the fifth thoracic vertebra. Fortunately, she regained near full power after surgical treatment of her spinal cyst.
\end{abstract}

\section{Keywords}

\section{Mediastinal Hydatid Cyst; Nuerogenic Tumor; Paraplegia}

\section{Introduction}

Hydatid disease is caused by Echinococcus granulosus parasite. It is an endemic disease; particularly in many Mediterranean countries. The liver and the lungs are most frequently involved [1]. Bone involvement is reported in $1 \%$ - $2 \%$ of the cases and about $50 \%$ of those are seen in the spine [2]. The first hydatid disease in spine was reported in 1807 by Chaussier [3]. Its clinical characteristics, diagnosis and therapy are still not definitive [3]. Spinal hydatid disease manifests itself through symptoms and signs related to compression of the cysts on other structures which has no specific pathognomonic symptoms or signs. The disease presents with radiculopathy, myelopathy and/or localized pain and tenderness owing to destructive bone lesions, pathological fracture and consequent cord compression [4]. X-ray appearance and computed tomographic scan findings of spinal echinococcosis are nonspecific and sometime misleading. MRI is a very sensitive diagnostic tool for hydatid cyst of spine [3]. 


\section{Case Report}

A 40-year-old lady referred to us suffering from painful spastic weakness of lower limbs, her condition started 6 months earlier to admission and she became paraplegic for last 4 months with loss of bladder and bowel control. She had L4, 5 lumber laminectomy 4 months earlier as she was diagnosed as lumber prolapsed intervertebral disc. Physical examination revealed bilateral lower limb weakness; power grade was zero, sensory loss from the level of D10, absence of all lower limb reflexes, distal pulses were positive. Per-rectal examination showed decreased anal tone. Chest radiograph showed left hilar shadow (Figure 1), CT scan revealed posterior mediastinal dumbbell tumor extended to the spinal canal at the level of D5, with destruction of left transverse process, pedicle, and fifth rib (Figure 2), MRI showed posterior mediastinal cystic lesion (Figure 3), Through mini-thoracotomy incision, exploration of the pleural cavity was done, cystic lesion was found in the left para-vertebral gutter with features of hydatid cyst, after isolation by Povidone-iodinesoaked packs aspiration of the cyst done which revealed caseating material and large number of small daughter cysts. Intraoperative orthopedic surgeon consultation requested who performed decompression of the canal anteriorly with partial anterolateral corpectomy. Histopathological examination of the specimen confirmed hydatidosis. She was treated by 800 mg Albendazole daily and program of physiotherapy. Full power movements appeared in 4 months post-operatively and her last MRI showed normal dorsal spinal cord with partial distortion of the body of D8-9 (Figure 4).

\section{Discussion}

Vertebral hydatidosis is a silent, slowly progressive disease. Pain is an important presenting symptom. Neurological disorders occur after a latent period of several years [5] [6]. The diagnosis is considered if the patient lives in a geographic region where the infestation is known to occur [7]. Our case didn't show any sign of chest involvement clinically and posterior mediastinal mass was an accidental finding on chest radiography during diagnostic workup for her paraplegia.

In bone tissue, the larva behaves differently from soft tissue. It grows in direction of least resistance, infiltrating and damaging the bone like a tumor by exogenous vesiculation, then enlarged by endogenous vesiculation re-

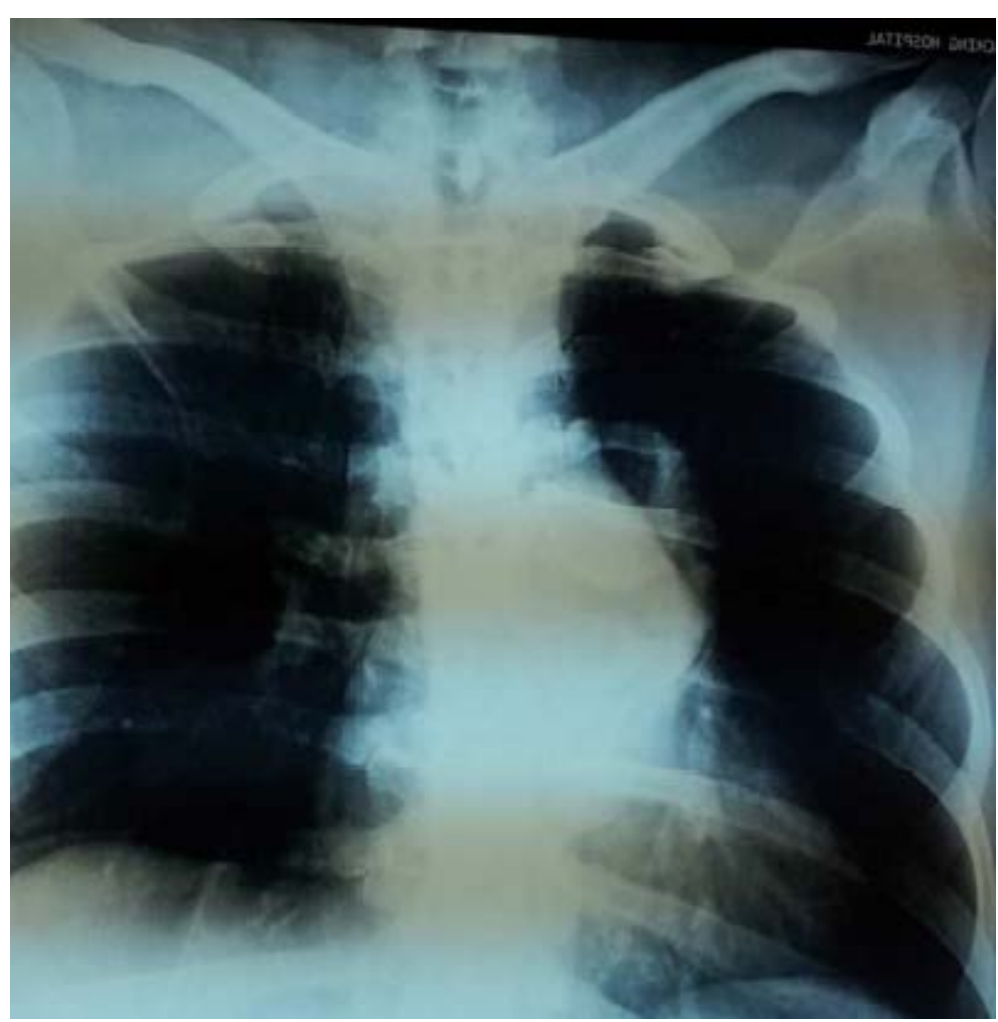

Figure 1. Ill-defined left hilar shadow. 


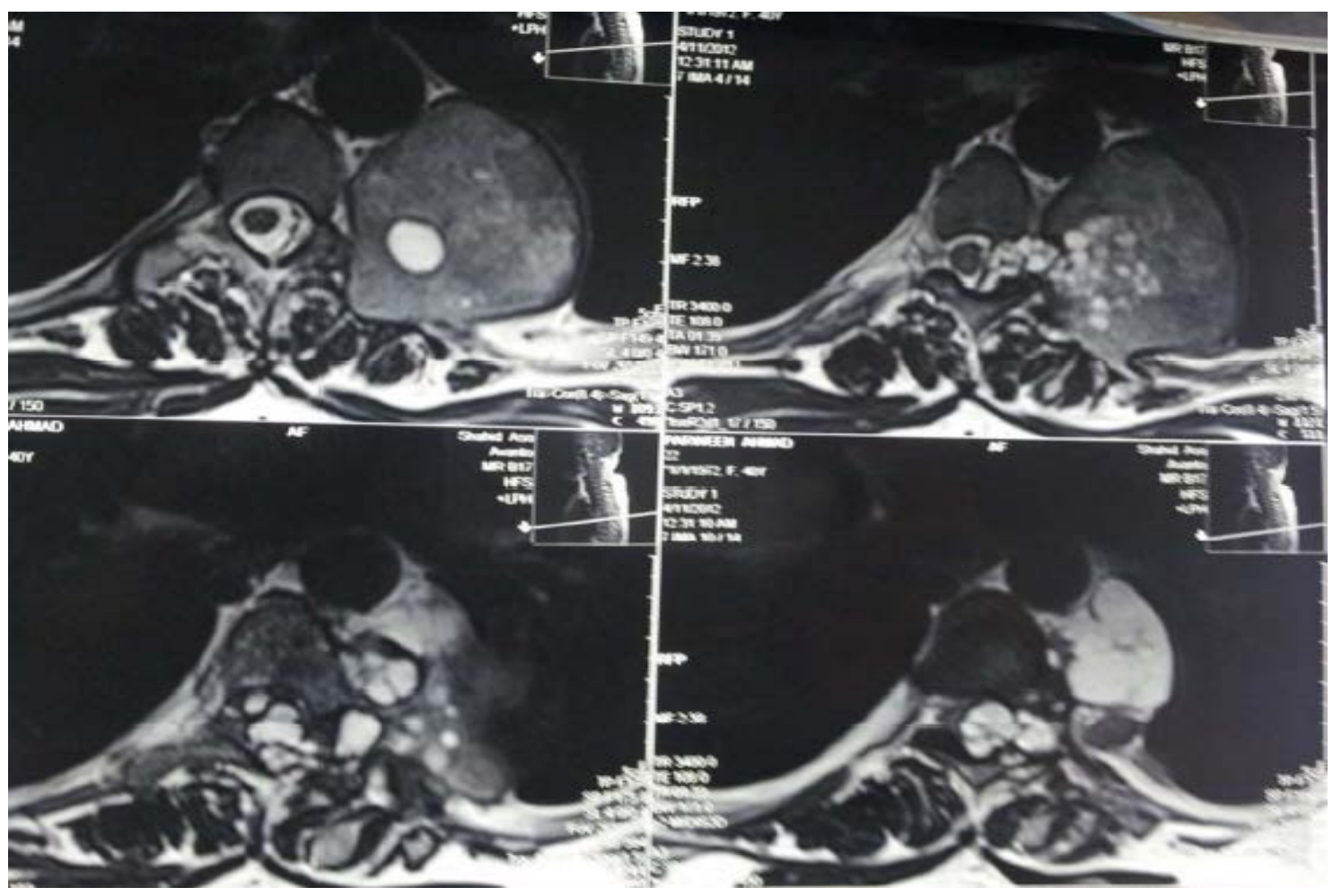

Figure 2. Posterior mediastinal mass with features of malignant neurogenic tumor.

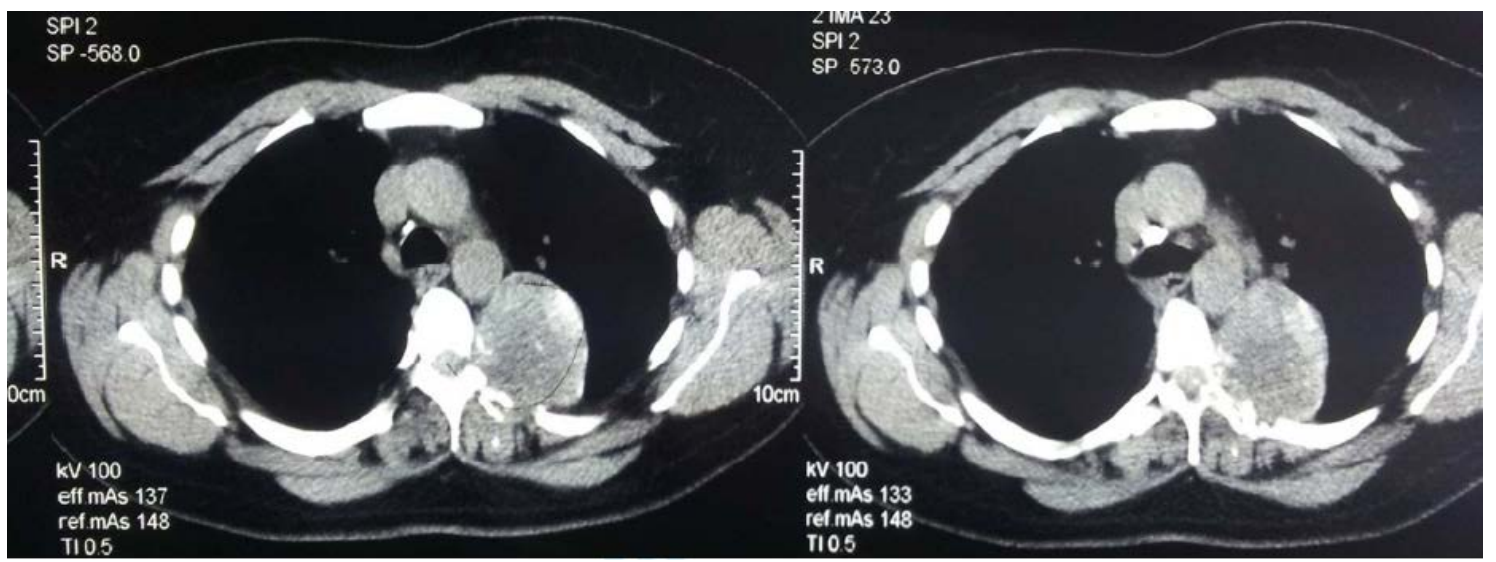

Figure 3. Posterior mediastinal dumbbell tumor extended to spinal canal at the level of D5-8, destruction of left transverse process, pedicle, and posterior part of fifth rib.

sulting in formation of daughter cyst [8]. Braithwaite and Lees classified the spinal hydatid as follow [9]:

- Primary intra medullary hydatid cyst.

- Intradural extra medullary hydatid cyst.

- Extradural intra spinal hydatid cyst.

- Hydatid disease of the vertebra.

- Paravertebral hydatid disease.

The first three groups of hydatidosis are rare, and only sporadic cases have been reported [9]. We can classify our case as type (III) who recovers from paraplegia and to our knowledge only few cases have been reported to regain full power [4] [6] [8]. Spinal involvement is believed to occur through vertebral-portal venous anastomosis or may be caused by encroaching pulmonary lesions. Rarely, the disease begins from the extradural region [9]. Most spinal hydatid cysts are thoracic (52\%), lumbar (37\%) and then the cervical and sacral levels [4]. The sur- 


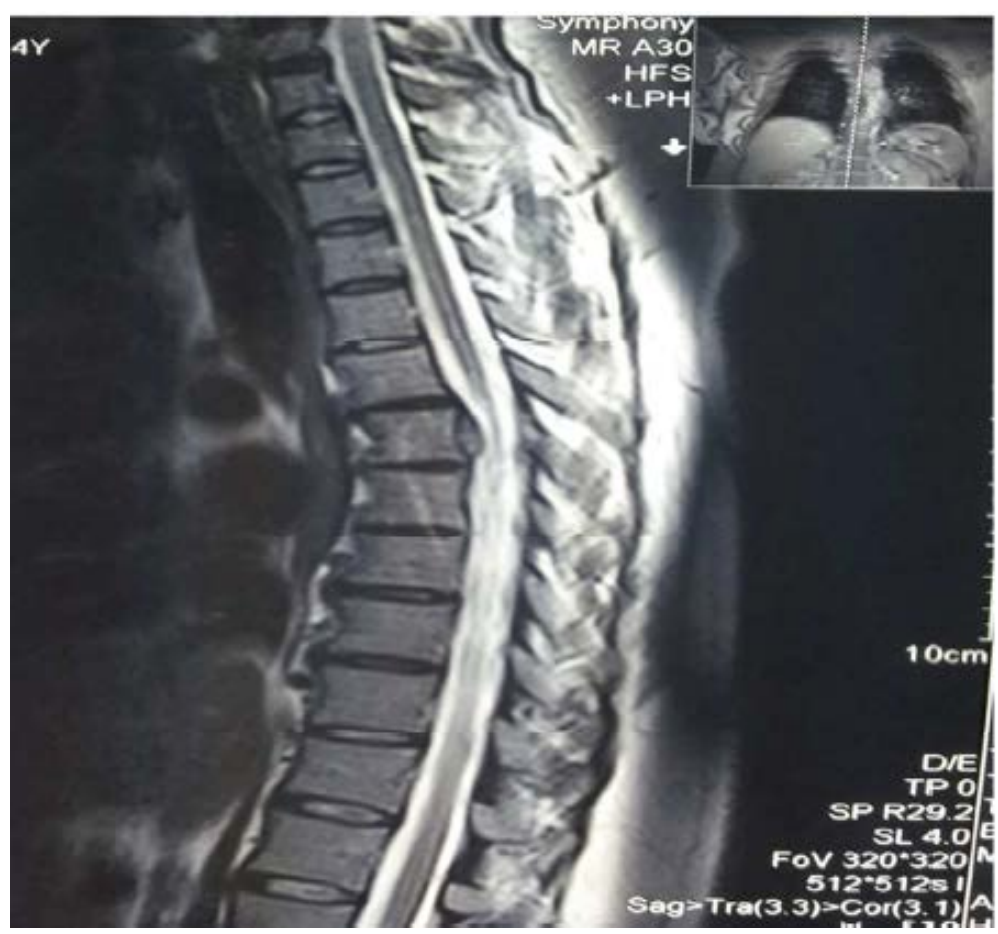

Figure 4. Normal dorsal cord with partial distortion of the body of D10.

gical treatment consists of removal of cyst contents without contamination followed by appropriate management of any remaining cavity. The surgical area might be irrigated with chemical agents in an attempt to kill scoleces [7]. Indications for chemotherapy include inoperable lesions unless it is not a harmful lesion, unwillingness of the patient to undergo surgery, and use as an adjunct to surgery. The combination of chemotherapy and surgical treatment has been found to be more efficient than surgical treatment alone [9]. Albendazole has been found to be better absorbed than Mebendazole and exhibits superior efficacy against helminthes [7].

Although extremely rare, intraspinal extradural thoracic hydatid cyst might be a reversible cause of paraplegia, sphincter dysfunction and sensory loss. Surgical decompression is the treatment of choice followed by postoperative chemotherapy and program of physiotherapy. Hydatid cyst may speculate all types of thoracic tumors, so high index of suspension should be considered in treating thoracic tumors in an endemic area.

\section{References}

[1] Awasthy, N. and Chand, K. (2005) Primary Hydatid Disease of the Spine: An Unusual Case. British Journal of Neurosurgery, 19, 425-427. http://dx.doi.org/10.1080/02688690500390359

[2] Zlitni, M., Kooli, M., Ezzaouia, K., Lebib, H. and Mestiri, M. (1996) Manifestations Osseuses des Parasitoses. Encycl Méd Chir, Appareil Locomoteur. Elsevier, Paris, 14021.

[3] Song, X.H., Ding, L.W. and Wen, H. (2007) Bone Hydatid Disease. Postgraduate Medical Journal, 83, 536-542. http://dx.doi.org/10.1136/pgmj.2007.057166

[4] Bhake, A. and Agrawal, A. (2010) Hydatid Disease of the Spine. Journal of Neurosciences in Rural Practice, 1, 61-62. http://dx.doi.org/10.4103/0976-3147.71715

[5] Dévé, F. (1948) L’Échinococcose Osseuse. Masson, Paris, 236.

[6] Mills, T.J. (1956) Paraplegia Due to Hydatid Disease. The Journal of Bone \& Joint Surgery, 38B, 884-891.

[7] Miloudi, G., Ali, A., Said, D., Driss, M., Abdelslam El, K. (2001) Primary Spinal Epidural Hydatid Cyst. Pan Arab Journal of Neurosurgery, 5, 50-52.

[8] Karray, S., Zlitni, M., Karray, M., Douik, M., Sliman, N., Sliman, N. and Litaiem, T., (1993) Extensive Vertebral Hydatidosis. Acta orthopedica Belgica, 59, 100-105.

[9] Brahwaite, P.A. and Lees, R.F. (1981) Vertebral Hydatid Disease. Radiological Assessment. Radiology, 140, $763-766$. 